\title{
Comparing the Effect of Methadone, Buprenorphine, and Opium Tincture on QTc Interval in Addicted Patients Undergoing Maintenance Therapy
}

Leila Hosseini ( $D$ Hosseini1115@yahoo.com)

North Khorasan University of Medical Sciences https://orcid.org/0000-0002-6617-9053

Malihe Seyedi

North Khorasan University of Medical Sciences

Seyyed Kaveh Hojjat

North Khorasan University of Medical Sciences

Nahid Rezaeian

Rajaie Cardiovascular Medical and Research Center

Mina Norouzi Khalili

North Khorasan University of Medical Sciences

Fatemeh Zadehbagheri

Yasuj University of Medical Sciences

Sanaz Asadian

Rajaie Cardiovascular Medical and Research Center

Saeed Bakhtiarpour

Islamic Azad University Ahvaz Branch

\section{Research}

Keywords: Maintenance therapy, methadone, buprenorphine, opium tincture, QTc interval

Posted Date: April 22nd, 2020

DOI: https://doi.org/10.21203/rs.2.22683/v2

License: (c) (i) This work is licensed under a Creative Commons Attribution 4.0 International License.

Read Full License 
The authors have withdrawn this preprint from Research Square 